\title{
LOS SISTEMAS INSTITUCIONALES DE EVALUACIÓN DE LOS ESTUDIANTES (SIEE) ENTRE LA TEORÍA Y LA REALIDAD PRÁCTICA ${ }^{1}$
}

\author{
Por: Álvaro Torres Mesías ${ }^{2}$ - Ana Barrios Estrada ${ }^{3}$ - Ruth Pantoja Burbano ${ }^{4}$
}

\section{RESUMEN}

El artículo hace parte de la investigación denominada "Los Sistemas Institucionales de Evaluación de los Estudiantes (SIEE) en la Instituciones Educativas Oficiales de la zona andina del Departamento de Nariño 2011-2012" aprobada por el Sistema de Investigaciones de la Universidad de Nariño, mediante acuerdo No. 034 de marzo 28 de 2011, desarrollada por el Grupo de Investigación para el desarrollo de la Educación y la Pedagogía (GIDEP).

En este artículo se presentan los resultados del proyecto de investigación mencionado que tuvo entre sus propósitos: establecer las concepciones, acuerdos y prácticas institucionales, identificar las percepciones y actitudes que maestros, padres de familia y estudiantes tienen sobre el SIEE y derivar algunos referentes teórico- prácticos para formular un plan de mejoramiento de los procesos evaluativos.

1. Los autores del artículo son integrantes del Grupo de Investigación para el Desarrollo Educativo y Pedagógico, Categoría”D” COLCIENCIAS (2014), que tiene como una de sus líneas: la pedagogía, desde donde adelanta proyectos orientados al estudio de los procesos evaluativos en el departamento de Nariño para hacer propuestas innovadoras que aporten en esta área.

2. Doctor en Ciencias Pedagógicas, Instituto Superior Pedagógico "Enrique José Varona”. La Habana, Cuba. Profesor de la Facultad de Educación de la Universidad de Nariño.

3. Candidata a Doctora en Ciencias de la Educación, Universidad del Cauca-Rudecolombia. Profesora de la Facultad de Educción de la Universidad de Nariño.

4. Estudiante de Maestría en Educación, Facultad de Educación de la Universidad de Nariño. Profesora de la Facultad de Educción de la Universidad Mariana.

Fecha de recepción: 29 de noviembre de 2013 - Fecha de aprobación definitiva: 10 de marzo de 2014 
La investigación se desarrolló con un enfoque de investigación cualitativa de tipo evaluativa, bajo la metodología de Investigación- Acción, que se desplegó en cinco momentos. En cuanto a los resultados se encontró que los docentes y las instituciones educativas interpretaron la nueva normativa sobre evaluación, específicamente el Decreto 1290 de 2009 del Ministerio de Educación Nacional (MEN) por el cual se reglamenta la evaluación del aprendizaje y promoción de los estudiantes de los niveles de educación básica y media, como un mecanismo para mejorar el rendimiento académico con el retorno a la evaluación cuantitativa de resultados, lo que significaría un retroceso de 50 años en la historia de la evaluación en Colombia.

En consecuencia, la norma que expidió el Ministerio de Educación Nacional (MEN) no ha implicado un cambio conceptual, ni actitudinal, ni procedimental en las prácticas evaluativas de los docentes y obliga a pensar que éste debe ir acompañado de acciones concretas para avanzar desde los logros alcanzados en busca del mejoramiento de los procesos evaluativos.

Palabras clave: Sistema Institucional de Evaluación de Estudiantes (SIEE), percepciones, actitudes, prácticas.

Clasificación JEL: 121. 


\title{
INSTITUTIONAL SYSTEMS OF STUDENT EVALUATION BETWEEN THEORY AND PRACTICAL REALITY
}

\author{
By: Álvaro Torres Mesías - Ana Barrios Estrada - Ruth Pantoja Burbano
}

\begin{abstract}
The article is part of the research entitled "Institutional Systems of student evaluation (SIEE) in the Educational Institutions of the Andean Department of Nariño 2011-2012," approved by the system of research of the University of Nariño by means of agreement No. 034 of 28 March, developed by the Research Group for the Development of Education and Pedagogy (GIDEP).

This article presents the results of the above mentioned research project which proposes: to establish concepts, agreements and institutional practices in order to identify perceptions and attitudes that teachers, parents, and students have regarding the SIEE, and to derive some related theoretical - practical information in order to formulate a plan for the improvement of evaluation processes.

The research was developed with a qualitative research approach of evaluative nature, under the research - action methodology, which was deployed in five stages. The outcomes found that teachers and educational institutions interpreted the new rules of evaluation (Decree 12902009 MEN) as a mechanism for improving academic performance, with a return to a quantitative evaluation of results, which would mean a decline of 50 years in the history of evaluation in Colombia.

Consequently, the standard issued by the Ministry of National Education (MEN) has involved neither a conceptual, attitudinal, nor procedural change in its evaluative practices of teachers, which forces the thought that these must be accompanied by concrete actions in order to advance achievements that seek improvements to evaluation processes.
\end{abstract}

Key words: Institutional system of students evaluation, perceptions, attitudes, practices.

JEL Classification: 121. 


\section{INTRODUCCIÓN}

El sistema educativo colombiano, en estos últimos años, ha experimentado cambios en el campo de la evaluación de estudiantes debido a la puesta en práctica en las Instituciones Educativas (I.E.) Oficiales del departamento de Nariño, del Sistema Institucional de Evaluación Educativa (SIEE) establecido mediante Decreto 1290 de 2009 del MEN, con la intencionalidad de intervenir los procesos de evaluación para mejorar su calidad. En consecuencia, con dicho decreto se reglamenta la evaluación del aprendizaje y la promoción de los estudiantes en los diferentes niveles de educación básica y media, a través de criterios, estrategias, procesos, acciones; se establece la periodicidad y estructura de los informes de los estudiantes y los mecanismos de participación de la comunidad educativa en la construcción del SIEE, especialmente como un llamado a la autonomía de las I. E. (MEN, 2009).

Debido a que estas nuevas disposiciones se refieren a los diferentes aspectos de la evaluación, su aplicación provocó múltiples reacciones y dificultades, tanto en su comprensión como en su aplicación, es así que la pretensión de mejoramiento, asociada al sistema de evaluación implementado en las Instituciones Educativas, se redujo a la urgencia de evaluar cuantitativamente, desconociendo una vez más que evaluar es uno de los actos más auténticamente pedagógicos por estar insertado en el proceso formativo, por tanto, demanda la coherencia entre el enfoque de evaluación y de enseñanza.

Esta nueva realidad se constituye en la problemática objeto de estudio de la presente investigación que documenta los procesos, las prácticas y actitudes, que se suscitan en algunas Instituciones Educativas Oficiales del Departamento de Nariño estudiadas, por parte de los diferentes actores, con la puesta en marcha del SIEE (Decreto 1290 de 2009), desde el año 2010, hasta el año 2012, periodo en el que se inicia la implementación de la nueva normativa.

\section{METODOLOGÍA}

La investigación se desarrolló con un enfoque de investigación cualitativa de tipo evaluativa, bajo la metodología de Investigación-Acción, que se desplegó en cinco momentos de investigación relacionados entre sí, como se describe a continuación.

- Trabajo de campo. Acercamiento a la realidad: Implicó la aplicación de entrevistas y encuestas a docentes, estudiantes, padres y madres de familia. Además se realizó un análisis de contenido al documento escrito de SIEE, y a las técnicas e instrumentos que se aplican para la evaluación de estudiantes.

- Informe de análisis preliminar: Se realizó el acercamiento a la realidad, luego se hizo el análisis de la información recopilada que permitió establecer los aciertos, las tensiones y dificultades de la implementación del SIEE. 
- Fundamentación teórica: Permitió confirmar y sustentar los aciertos, las dificultades y tensiones detectadas que se fundamentaron con autores y obras que han tratado dichas situaciones.

- Devolución de la información: En este momento de la investigación y con miras a construir colectivamente la caracterización de la realidad y el referente teórico, se devolvió la información en el I Simposio de Evaluación del Aprendizaje, desarrollado durante los días 13, 14 y 15 de noviembre de 2013 en las Instalaciones de la Universidad de Nariño.

- Formulación del plan de mejoramiento de los procesos evaluativos. A partir de los hallazgos alcanzados en los anteriores momentos se elaboró un plan de mejoramiento para el SIEE.

\section{DISCUSIÓN DE RESULTADOS}

Se presenta a continuación la discusión de los resultados alcanzados en esta investigación que permitió el encuentro con la realidad de la evaluación en las instituciones educativas estudiadas, desde la mirada de los profesores, los estudiantes y los padres de familia a partir de la implementación del Decreto 1290 de 2009 que pretendía generar "mejores prácticas educativas, ambientes de aprendizaje y de evaluación, para apoyar los aprendizajes de los estudiantes” MEN ( 2009, p. 30), desde la autonomía de los colectivos de maestros trabajando con todos los actores de la comunidad educativa.

De igual forma, esta investigación abrió la posibilidad de reconocer los acuerdos y prácticas institucionales que se suscitaron en torno a la evaluación del aprendizaje de los estudiantes, que llaman la atención por la persistencia de concepciones y prácticas evaluativas asociadas a procesos de enseñanza y aprendizaje transmisionistas con la idea de evaluar para medir, calificar y mejorar el rendimiento académico.

\subsection{Percepciones y actitudes de los profesores}

Los maestros encuestados, en su mayoría, consideran que el SIEE reconoce su autonomía, no obstante, los maestros expresan una ambivalencia al referirse en el mismo porcentaje tanto a los sentimientos de ansiedad y preocupación por las exigencias que demanda el SIEE, como al agrado ante esta experiencia, como se puede apreciar en los resultados de la tabla 1.

Estos resultados evidencian las dificultades para comprender el SIEE y la nueva normativa debido a que son directrices que pretenden convertirse en la principal herramienta pedagógica para el mejoramiento de la calidad educativa y orientar el cambio de uno de los procesos más complejos y que presenta mayor resistencia, como es la evaluación. Aunque el MEN (2009) evidencia el proceso participativo de construcción colectiva y la posterior receptividad positiva de la ciudadanía sobre el decreto, los maestros encuestados manifiestan sentimientos y actitudes 
diversas, debido a las nuevas demandas que se le hace al maestro, en el sentido de implementar estrategias novedosas en el campo de la evaluación porque el decreto "es una clara invitación a los colectivos de maestros y a las instituciones educativas" (p. 30), para que trabajen con todos los actores de la comunidad educativa, a fin de "consolidar mejores prácticas educativas, ambientes de aprendizaje y de evaluación, para apoyar los aprendizajes de los estudiantes” (p. 30). La dimensión de estas demandas puede estar relacionada con la presencia de desconcierto, ansiedad y preocupación en los maestros encuestados, al encontrar que "la tarea de construir un sistema institucional, es un proceso amplio, complejo, de mucha responsabilidad y gran relevancia en cualquier institución escolar” (MEN, 2009:34).

Tabla 1: Percepciones y actitudes de los docentes sobre las características de la evaluación

\begin{tabular}{|l|c|c|c|c|c|c|}
\hline \multicolumn{1}{|c|}{ ASPECTOS A CONSIDERAR } & TA & PA & NA/ND & PD & TD & NR \\
\hline $\begin{array}{l}\text { Siento que la creación del sistema institu- } \\
\text { cional de evaluación de los estudiantes nos } \\
\text { generó desconcierto y ansiedad. }\end{array}$ & $31,1 \%$ & $45,9 \%$ & $4,9 \%$ & $8,2 \%$ & $8,2 \%$ & $1,6 \%$ \\
\hline $\begin{array}{l}\text { Considero que el sistema institucional de } \\
\text { evaluación de los estudiantes reconoce } \\
\text { nuestra autonomía. }\end{array}$ & $39,3 \%$ & $36,1 \%$ & $11,5 \%$ & $9,8 \%$ & $3,3 \%$ & $0,0 \%$ \\
\hline $\begin{array}{l}\text { Me preocupan las exigencias del sistema ins- } \\
\text { titucional de evaluación de los estudiantes. }\end{array}$ & $26,2 \%$ & $29,5 \%$ & $18,0 \%$ & $16,4 \%$ & $9,8 \%$ & $0,0 \%$ \\
\hline $\begin{array}{l}\text { Me agrada la experiencia con el sistema ins- } \\
\text { titucional de evaluación de los estudiantes. }\end{array}$ & $31,1 \%$ & $39,3 \%$ & $21,3 \%$ & $4,9 \%$ & $3,3 \%$ & $0,0 \%$ \\
\hline
\end{tabular}

Fuente: Esta investigación

Sin embargo, otros maestros consultados expresan su agrado con esta experiencia que demanda el ejercicio de la autonomía escolar, establecida desde el año 1994 en la Ley General de Educación, y según el MEN (2009: 34) se trata de abandonar la idea de "tener un único modelo y sistema de evaluación de los estudiantes para todo el país". En consecuencia, se hace necesario dialogar y llegar consensos con toda la comunidad educativa, conocer la cultura escolar, los contextos, además implica el estudio, reflexión, análisis, "para no generar efectos contrarios a los previstos o que la reforma no vaya más allá de la introducción de conceptos novedosos que no cambian en nada las prácticas tradicionales" . Es por eso que la tarea de construir un sistema institucional de evaluación, es un proceso amplio, complejo, de mucha responsabilidad y gran relevancia para toda institución educativa (MEN, 2009:34).

Por otra parte, los maestros encuestados, en sus percepciones reconocen que el SIEE contribuye al mejoramiento del rendimiento académico de los estudiantes (Tabla 2). 
Tabla 2: Percepciones de los docentes el aporte del SIEE al rendimiento académico de los estudiantes

\begin{tabular}{|c|c|c|c|c|c|c|}
\hline ASPECTOS A CONSIDERAR & TA & PA & NA/ND & PD & TD & NR \\
\hline $\begin{array}{l}\text { Reconozco la contribución al mejoramiento del } \\
\text { rendimiento académico por parte del sistema } \\
\text { institucional de evaluación de los estudiantes. }\end{array}$ & $34,4 \%$ & $44,3 \%$ & $6,6 \%$ & $11,5 \%$ & $3,3 \%$ & $0,0 \%$ \\
\hline
\end{tabular}

Fuente: Esta investigación

$\mathrm{Al}$ respecto se puede inferir claramente de los resultados que los docentes han establecido una correspondencia entre el mejoramiento del rendimiento académico y la implementación del SIEE, este es un hecho que puede estar sustentado en la influencia que tiene el peso de la tradición, la resistencia al cambio que se encuentra instalada en los actores escolares, es por eso que se toma el SIEE como nueva expresión, pero se mantiene la preocupación por el rendimiento académico, y con esta se sostienen las estructuras académicas, administrativas acríticas, el divorcio entre teorías y práctica y las prácticas transmisionistas.

En consecuencia, esta es una evidencia de la incomprensión del sentido y los propósitos de la evaluación en el decreto 1290/09 y la implementación del SIEE que no se concibió en función del rendimiento académico, si no para "recoger información sobre aprendizajes significativos, críticos, autónomos, cooperativos, sociales y solidarios" (MEN, 2009: 29), al concebir una evaluación formativa que permita a los estudiantes poner en práctica sus conocimientos, defender sus ideas, exponer sus razones, saberes, dudas, ignorancias e inseguridades con la intención de superarlas (MEN, 2009).

De otro lado, los maestros expresan divergencias en cuanto a su preparación para implementar el SIEE, sobre su imposición y la aceptación de directrices, que se manifiestan en pensamientos y sentimientos disímiles que oscilan entre parcialmente de acuerdo y totalmente en desacuerdo, como se muestra en la tabla 3.

Tabla 3: Percepciones y actitudes de los docentes sobre su preparación para implementar el SIEE

\begin{tabular}{|c|c|c|c|c|c|c|}
\hline \multicolumn{1}{|c|}{ ASPECTOS A CONSIDERAR } & TA & PA & NA/ND & PD & TD & NR \\
\hline $\begin{array}{l}\text { Pienso que no estábamos preparados para im- } \\
\text { plementar el sistema institucional de evalua- } \\
\text { ción de los estudiantes. }\end{array}$ & $8,2 \%$ & $36,1 \%$ & $14,8 \%$ & $9,8 \%$ & $29,5 \%$ & $1,6 \%$ \\
\hline $\begin{array}{l}\text { Me disgusta que nos impongan el sistema ins- } \\
\text { titucional de evaluación de los estudiantes. }\end{array}$ & $16,4 \%$ & $29,5 \%$ & $14,8 \%$ & $13,1 \%$ & $24,6 \%$ & $1,6 \%$ \\
\hline $\begin{array}{l}\text { Acepto las directrices del sistema institucional } \\
\text { de evaluación de los estudiantes. }\end{array}$ & $47,5 \%$ & $27,9 \%$ & $8,2 \%$ & $9,8 \%$ & $6,6 \%$ & $0,0 \%$ \\
\hline
\end{tabular}

Fuente: Esta investigación 
Se aprecia que en el pensamiento de los maestros consultados se identifican desacuerdos en cuanto a su preparación para implementar el SIEE, esto se debe en gran medida a las diferentes interpretaciones que se le ha dado al sentido y los propósitos de la evaluación en el decreto 1290/09 y la implementación del SIEE, como se explicó en los párrafos anteriores, si dicha puesta en marcha está asociada solamente al rendimiento académico, no se requiere mayor preparación por parte de los maestros; pero si se trata de una evaluación formativa para recoger información sobre aprendizajes significativos, críticos, autónomos, entre otros, se debe reconocer que los maestros no estaban preparados.

Otra manifestación de los maestros encuestados hace evidente las divergencias en cuanto a la imposición del SIEE, que a la mayoría les disgusta, otros están de acuerdo, pero en todo caso consideran que hay una imposición, porque no se sienten participes de este cambio que lo asumen como otra directriz que viene desde arriba, en este caso del Ministerio de Educación Nacional.

Llama la atención el porcentaje de maestros indiferentes, tanto a la preparación para implementar el SIEE como a su imposición, que puede ser uno de los orígenes de las interpretaciones inadecuadas, de los reduccionismos y las actitudes de resistencia que, aparentemente, hacen algunas adecuaciones pero en el fondo esperan que nada cambie en las prácticas de evaluación, situación que se puede ver fortalecida con el alto porcentaje de maestros que dicen aceptar las directrices del SIEE, probablemente de manera acrítica y por temor a la sanción, con lo cual toda reforma e innovación quedan reducidas a buenas intenciones que no se pueden concretar, porque se requiere que el maestro y todos los actores escolares hagan una ruptura con la idea de evaluar para medir, calificar y mejorar el rendimiento académico, que les permita avanzar en nuevas formas de pensar y hacer la evaluación formativa de los aprendizajes desde enfoques incluyentes, integradores, participativos para el desarrollo de las competencias en coherencia con el proyecto educativo institucional, los contextos y que se legitime con la participación de la comunidad educativa.

\subsection{Percepciones y actitudes de los profesores y los estudiantes}

En cuanto a la percepción de profesores y estudiantes sobre la evaluación de las características personales por parte del SIEE se encuentran diferentes manifestaciones como se muestra en los resultados de las tablas 4 y 5 .

Tabla 4: Percepciones de los docentes sobre la evaluación de las características personales por parte del SIEE

\begin{tabular}{|l|r|r|r|r|r|l|}
\hline \multicolumn{1}{|c|}{ ASPECTOS A CONSIDERAR } & S & CS & AV & CN & N & NR \\
\hline $\begin{array}{l}\text { En su colegio se evalúan las características perso- } \\
\text { nales de los estudiantes (colaborador, desenvuel- } \\
\text { to, responsable, ordenado, trabajador, puntual). }\end{array}$ & $72 \%$ & $18 \%$ & $9 \%$ & $1 \%$ & $0 \%$ & $0 \%$ \\
\hline $\begin{array}{l}\text { En las evaluaciones de su colegio se reconocen } \\
\text { los intereses y necesidades de los estudiantes. }\end{array}$ & $35 \%$ & $43 \%$ & $22 \%$ & $0 \%$ & $0 \%$ & $0 \%$ \\
\hline
\end{tabular}

Fuente: Esta investigación 
Tabla 5: Percepciones de los estudiantes sobre la evaluación de las características personales por parte del SIEE

\begin{tabular}{|l|c|c|c|c|c|c|}
\hline \multicolumn{1}{|c|}{ ASPECTOS A CONSIDERAR } & S & CS & AV & CN & N & NR \\
\hline $\begin{array}{l}\text { En el colegio te evalúan de manera integral en } \\
\text { todos los aspectos académicos, personales y so- } \\
\text { ciales. }\end{array}$ & $47 \%$ & $35,9 \%$ & $9,9 \%$ & $5,3 \%$ & $2 \%$ & $0,0 \%$ \\
\hline $\begin{array}{l}\text { El colegio te pide que cumplas con los compro- } \\
\text { misos académicos y de convivencia. }\end{array}$ & $67 \%$ & $11,5 \%$ & $13,7 \%$ & $6,1 \%$ & $2 \%$ & $0,0 \%$ \\
\hline
\end{tabular}

Fuente: Esta investigación

Donde se evidencia que según la mayoría de profesores si se evalúan las características personales de los estudiantes, como parte de su formación integral y el desarrollo de las competencias, referidas especialmente a las actitudes, intereses y necesidades, por su parte los estudiantes, en un menor porcentaje, también están de acuerdo que en su I.E. se realiza la evaluación con estas características, sin embargo, llama la atención que algunos estudiantes consideran lo contrario.

Estos resultados muestran el avance hacia nuevas formas de evaluación que superan la preocupación exclusiva por el rendimiento académico y la calificación del dominio de contenidos como resultado de la transmisión de conocimientos. De esta manera, se muestra un acercamiento a los propósitos y características que se plantean en el SIEE en el sentido de "identificar las características personales, intereses, ritmos de desarrollo y estilos de aprendizaje para valorar sus avances; proporcionar información básica para consolidar o reorientar los procesos educativos relacionados con el desarrollo integral del estudiante” (MEN, 2009: 21, 29, 30).

Por otra parte, los docentes encuestados en su gran mayoría tienen conocimiento sobre la evaluación de los aprendizajes de sus estudiantes y a los avances que obtienen en dicho proceso, como se presenta en los resultados de la tabla 6 .

Tabla 6: Percepciones de los docentes sobre la evaluación de los aprendizajes

\begin{tabular}{|l|c|c|c|c|c|c|}
\hline \multicolumn{1}{|c|}{ ASPECTOS A CONSIDERAR } & S & CS & AV & CN & N & NR \\
\hline $\begin{array}{l}\text { Con las evaluaciones que Usted realiza puede iden- } \\
\text { tificar la forma como aprenden los estudiantes. }\end{array}$ & $56 \%$ & $37 \%$ & $7 \%$ & $0 \%$ & $0 \%$ & $0 \%$ \\
\hline $\begin{array}{l}\text { Las evaluaciones que Usted realiza le permiten sa- } \\
\text { ber si el estudiante aprendió y como avanza en su } \\
\text { aprendizaje. }\end{array}$ & $66 \%$ & $26 \%$ & $7 \%$ & $0 \%$ & $0 \%$ & $0 \%$ \\
\hline
\end{tabular}

Fuente: Esta investigación 
Dichos resultados indican que las evaluaciones realizadas por los profesores están orientadas a identificar la forma como aprenden los estudiantes, el aprendizaje que logran y los avances. Este es una evidencia positiva en el proceso de evaluación que permite al docente identificar los diferentes ritmos de aprendizaje de los estudiantes, los progresos y las dificultades para informar a tiempo al estudiante e implementar estrategias que le permitan superar las deficiencias que se manifiestan en el proceso de enseñanza y aprendizaje, de esta manera se promueve la integración entre estos procesos que son importantes desde la evaluación formativa, que es concebida como una actividad continua y no sólo al final de un tema o unidad como si fuera independiente de la formación.

Por su parte, los estudiantes manifiestan que cuentan con información sobre los instrumentos con los que son evaluados, también conocen sus calificaciones y reciben acompañamiento por parte de los docentes y la I.E. que exige el cumplimiento de compromisos, como se presentan en los resultados de la tabla 7.

Tabla 7: Percepciones de los estudiantes sobre la información y acompañamiento en el proceso de evaluación

\begin{tabular}{|l|c|c|c|c|c|c|}
\hline \multicolumn{1}{|c|}{ ASPECTOS A CONSIDERAR } & S & CS & AV & CN & N & NR \\
\hline $\begin{array}{l}\text { Te informan desde el inicio del año escolar sobre } \\
\text { la evaluación que te hacen, cómo y con qué? } \\
\text { (talleres, trabajos de consulta, observaciones, } \\
\text { participaciones, exámenes, etc.). }\end{array}$ & $61 \%$ & $21,4 \%$ & $13,7 \%$ & $3,1 \%$ & $1 \%$ & $0,0 \%$ \\
\hline $\begin{array}{l}\text { Puedes conocer tus calificaciones a tiempo y re- } \\
\text { cibir respuestas a tus inquietudes y solicitudes. }\end{array}$ & $27 \%$ & $41,2 \%$ & $23,7 \%$ & $6,9 \%$ & $1 \%$ & $0,0 \%$ \\
\hline $\begin{array}{l}\text { Recibes el acompañamiento de los docentes } \\
\text { cuando tienes problemas en alguna materia. }\end{array}$ & $31 \%$ & $26,7 \%$ & $31,3 \%$ & $7,6 \%$ & $3 \%$ & $0,0 \%$ \\
\hline $\begin{array}{l}\text { El colegio te exige que cumplas con las recomen- } \\
\text { daciones y compromisos para superar los proble- } \\
\text { mas en alguna materia. }\end{array}$ & $56 \%$ & $17,6 \%$ & $16,0 \%$ & $9,2 \%$ & $1 \%$ & $0,0 \%$ \\
\hline
\end{tabular}

Fuente: Esta investigación

Se aprecia un alto porcentaje de estudiantes que reconocen como los docentes desde el inicio del año escolar les informan sobre las formas de evaluación y los diferentes instrumentos con los que evaluarán sus aprendizajes, de esta manera se ve reflejado que los criterios de evaluación son claros y negociados entre profesores y estudiantes que garantizan ecuanimidad y equidad en el proceso de evaluación.

En un menor porcentaje los estudiantes manifiestan que conocen a tiempo las calificaciones y reciben acompañamiento por parte de los docentes cuando tienen 
inquietudes, dificultades y solicitudes relacionadas con el proceso de evaluación. Estos resultados permiten inferir que se están realizando esfuerzos para ofrecer un acompañamiento con el fin de que el estudiante logre nivelarse en cuanto a las competencias y estándares establecidos para determinado grado. De esta manera el docente debe generar estrategias innovadoras para motivar y guiar a los estudiantes en sus procesos complementarios de aprendizaje apoyados en el acompañamiento que realicen los padres de familia desde sus hogares, a fin de superar progresivamente sus dificultades para llegar a buen término al finalizar su año escolar.

Los profesores explicitan que los propósitos de la evaluación están orientados a determinar la promoción de los estudiantes y a partir de los resultados de evaluación se formulan planes de mejoramiento, como se ve en los resultados de la tabla 8.

Tabla 8: Percepción de los profesores sobre la promoción y los planes de mejoramiento

\begin{tabular}{|c|c|c|c|c|c|c|}
\hline ASPECTOS A CONSIDERAR & S & CS & AV & CN & N & NR \\
\hline $\begin{array}{l}\text { Los propósitos de la evaluación en su } \\
\text { colegio permiten determinar la pro- } \\
\text { moción y/o promoción anticipada de } \\
\text { los estudiantes. }\end{array}$ & $44 \%$ & $21 \%$ & $18 \%$ & $7 \%$ & $7 \%$ & $3 \%$ \\
\hline $\begin{array}{l}\text { En su colegio se formula un plan de } \\
\text { mejoramiento institucional como re- } \\
\text { sultado de la evaluación }\end{array}$ & $66 \%$ & $21 \%$ & $12 \%$ & $1 \%$ & $0 \%$ & $0 \%$ \\
\hline
\end{tabular}

Fuente: Esta investigación

Estos hallazgos demuestran que los profesores y las instituciones educativas han acogido los planteamientos del decreto 1290/09 en cuanto a la autonomía para determinar la promoción de los estudiantes y el uso de los resultados de la evaluación para el ajuste e implementación del plan de mejoramiento institucional; en busca de la calidad, sería conveniente hacer un seguimiento a estas iniciativas para conocer los aportes y su sostenibilidad y evolución en el tiempo.

\subsection{Percepciones de los padres de familia sobre el proceso de evaluación}

Sabemos que los padres de familia son los principales responsables de la formación de sus hijos y que deben participar de manera activa en el proceso a través del conocimiento del sistema institucional de evaluación en cuanto a los criterios, instrumentos, procesos y promoción de los estudiantes; no solo deben recibir los informes sino plantear inquietudes y sugerencias que lleven a fortalecer la formación de sus hijos y ayudarlos a superar sus dificultades. 
Los padres de familia manifestaron que la institución educativa les comunica y les da participación en el proceso de evaluación de sus hijos como se muestra en los resultados de la tabla 9.

Tabla 9: Percepción de los padres de familia sobre la comunicación que tienen con la I. E.

\begin{tabular}{|l|c|c|c|c|c|c|}
\hline \multicolumn{1}{|c|}{ ASPECTOS A CONSIDERAR } & S & CS & AV & CN & N & NR \\
\hline $\begin{array}{l}\text { El colegio le informa a usted sobre la evaluación } \\
\text { que realizan sus hijos? }\end{array}$ & $61,2 \%$ & $13,8 \%$ & $19,8 \%$ & $2,6 \%$ & $2,6 \%$ & $0,0 \%$ \\
\hline $\begin{array}{l}\text { El colegio le dice la forma como realizan la eva- } \\
\text { luación de sus hijos? }\end{array}$ & $56,0 \%$ & $13,8 \%$ & $14,7 \%$ & $6,9 \%$ & $8,6 \%$ & $0,0 \%$ \\
\hline $\begin{array}{l}\text { A usted como padre de familia lo invitan a parti- } \\
\text { cipar en el gobierno escolar para decidir sobre la } \\
\text { evaluación y promoción de sus hijos? }\end{array}$ & $53,4 \%$ & $11,2 \%$ & $10,3 \%$ & $4,3 \%$ & $20,7 \%$ & $0,0 \%$ \\
\hline $\begin{array}{l}\text { El Colegio le exige que esté atento a la evalua- } \\
\text { ción que realizan sus hijos? }\end{array}$ & $69,8 \%$ & $13,2 \%$ & $10,4 \%$ & $0,9 \%$ & $5,7 \%$ & $0,0 \%$ \\
\hline $\begin{array}{l}\text { El colegio explica los resultados de los boletines } \\
\text { que entrega en cada periodo? }\end{array}$ & $85,3 \%$ & $7,8 \%$ & $0,9 \%$ & $1,7 \%$ & $4,3 \%$ & $0,0 \%$ \\
\hline
\end{tabular}

Fuente: Esta investigación

Dichos resultados, a nivel general, muestran que la comunicación entre padres de familia y la institución educativa es buena, porque les informa sobre la evaluación que realizan a sus hijos, sobre las estrategias y actividades que los docentes desarrollan, también los invitan a formar parte del gobierno escolar, les exigen que estén atentos a la evaluación que realizan sus hijos y les explican los resultados de la evaluación contenida en los boletines que se les entrega cada periodo. Sin embargo, existe un porcentaje de padres de familia que aún no están enterados y desconocen el proceso que se lleva a cabo al interior de las instituciones, en cuanto a la evaluación, por eso es necesario que las I. E. propongan otro tipo de estrategias para llevar la información de los padres y lograr que hagan parte del proceso y sean considerados los padres de familia "agentes activos y participativos en el proceso evaluativo de sus hijos" (MEN, 2009:63), de tal manera que asuman un actitud comprensiva en el acompañamiento durante la vida escolar de sus hijos, que generen confianza y apoyo a las dificultades que puedan tener en el transcurso de este periodo.

Esta idea esta respaldada por el decreto 1290 mediante el cual se crea el sistema institucional de evaluación de estudiantes, donde abre un nuevo escenario de participación a los padres de familia, dándoles posibilidades de "intervenir en la construcción de los SIEE, además de hacer el respectivo seguimiento a los procesos de aprendizaje de sus hijos" MEN (2009: 64). 
De otro lado, los padres de familia, manifiestan interés y compromiso con el proceso de evaluación que desarrollan sus hijos, de acuerdo a lo que se muestra en la tabla 10.

Tabla 10: Percepción de los padres de familia sobre la comunicación que tienen con la Institución Educativa

\begin{tabular}{|l|c|c|c|c|c|c|}
\hline \multicolumn{1}{|c|}{ ASPECTOS A CONSIDERAR } & S & CS & AV & CN & N & NR \\
\hline $\begin{array}{l}\text { ¿Usted está pendiente de las evaluaciones } \\
\text { que les hacen a sus hijos en el colegio? }\end{array}$ & $62,9 \%$ & $18,1 \%$ & $12,1 \%$ & $3,4 \%$ & $2,6 \%$ & $0,9 \%$ \\
\hline $\begin{array}{l}\text { ¿Usted asiste a las reuniones de entrega } \\
\text { de boletines en cada período? }\end{array}$ & $89,7 \%$ & $7,8 \%$ & $2,6 \%$ & $0,0 \%$ & $0,0 \%$ & $0,0 \%$ \\
\hline $\begin{array}{l}\text { ¿Usted visita al profesor para averiguar } \\
\text { sobre el rendimiento académico de sus } \\
\text { hijos? }\end{array}$ & $46,6 \%$ & $21,6 \%$ & $19,8 \%$ & $4,3 \%$ & $6,9 \%$ & $0,9 \%$ \\
\hline
\end{tabular}

Fuente: Esta investigación

Los resultados muestran que los padres de familia están comprometidos con el proceso de evaluación de sus hijos, manifiestan estar pendientes de las evaluaciones, asistir a las reuniones de entrega de boletines y visitar a los docentes para averiguar sobre el rendimiento académico de sus hijos. Como se había mencionado anteriormente, es de vital importancia la participación de los padres de familia en el proceso de evaluación, y hace parte de su responsabilidad como padres, interesarse en este proceso, debido a que las decisiones que se tomen al interior del establecimiento educativo afectan positiva o negativamente la vida de sus hijos, por lo que es indispensable estar informado sobre el "desarrollo del PEI, los resultados de las evaluaciones internas y externas, la auto evaluación institucional y la ejecución de planes de mejoramiento" MEN (2009:64), la participación de las reuniones y la comprensión de los resultados de la evaluación, para que pueda convertirse en un apoyo para el docente en favor del proceso educativo de sus hijos.

\subsection{Acuerdos y prácticas institucionales en torno a la evaluación del aprendizaje de los estudiantes}

En este acápite se muestra los resultados de investigación que responden al interrogante ¿̇uáles son las condiciones teóricas, metodológicas y actitudinales de maestros en ejercicio, en torno a su práctica pedagógica evaluativa actual? Estos resultados se organizan en categorías y subcategorías emergentes de los testimonios escritos por los maestros consultados que se analizan a partir de sus relaciones en redes semánticas que abren la posibilidad de hacer comentarios desde la teoría y la interpretación de los investigadores, como se presenta a continuación: 


\section{Cuadro 1: Análisis y comentarios de las concepciones sobre evaluación de los maestros}

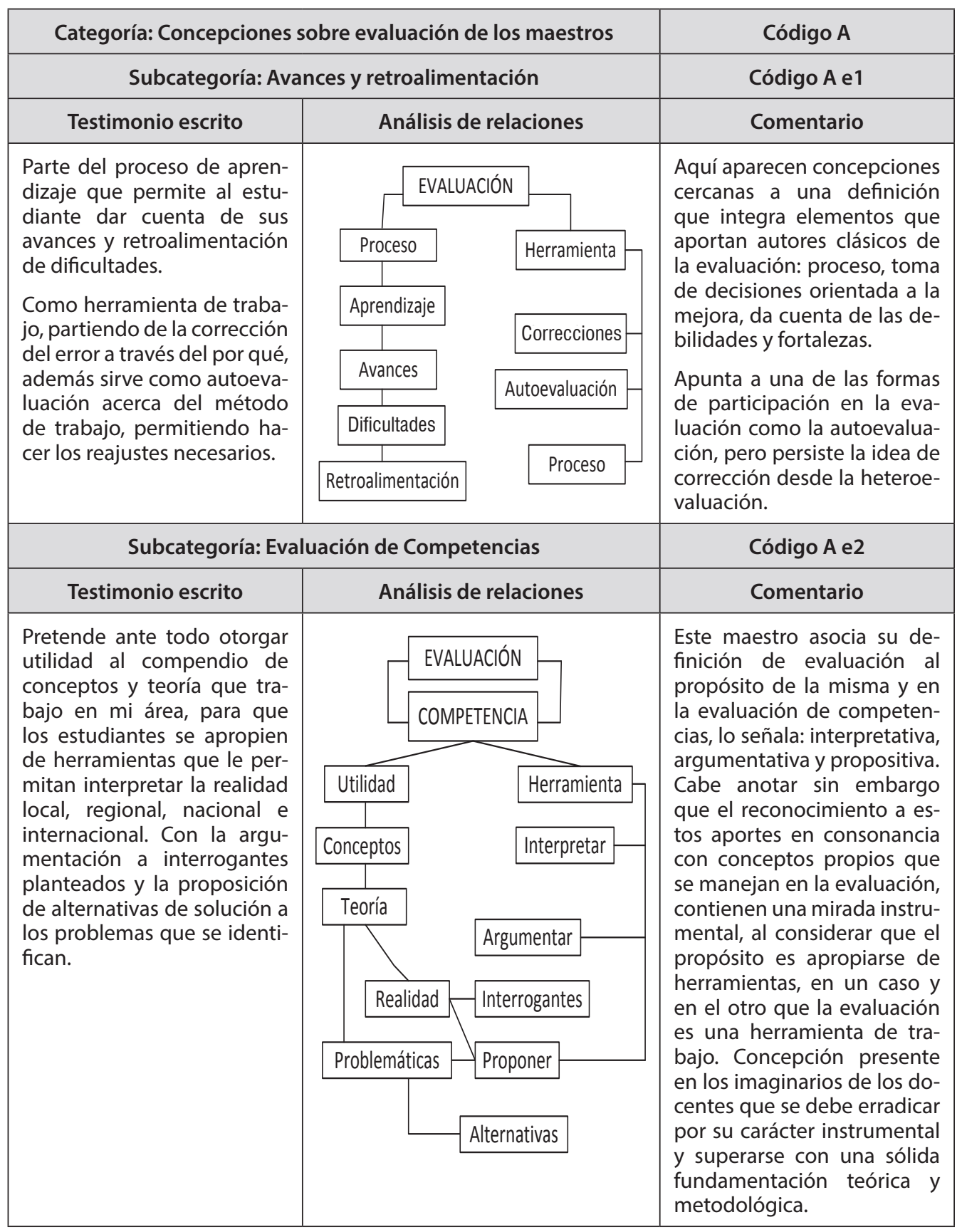




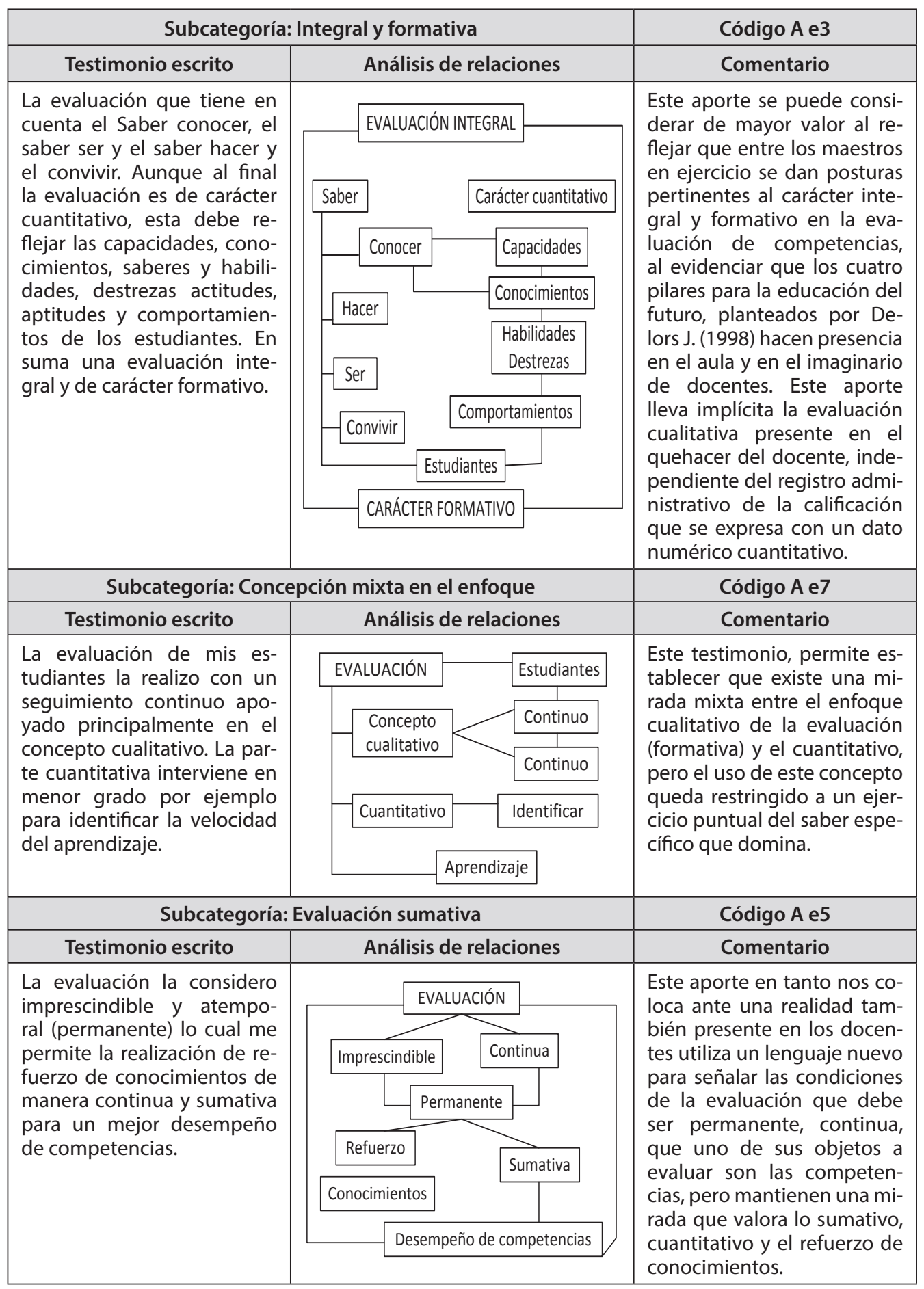




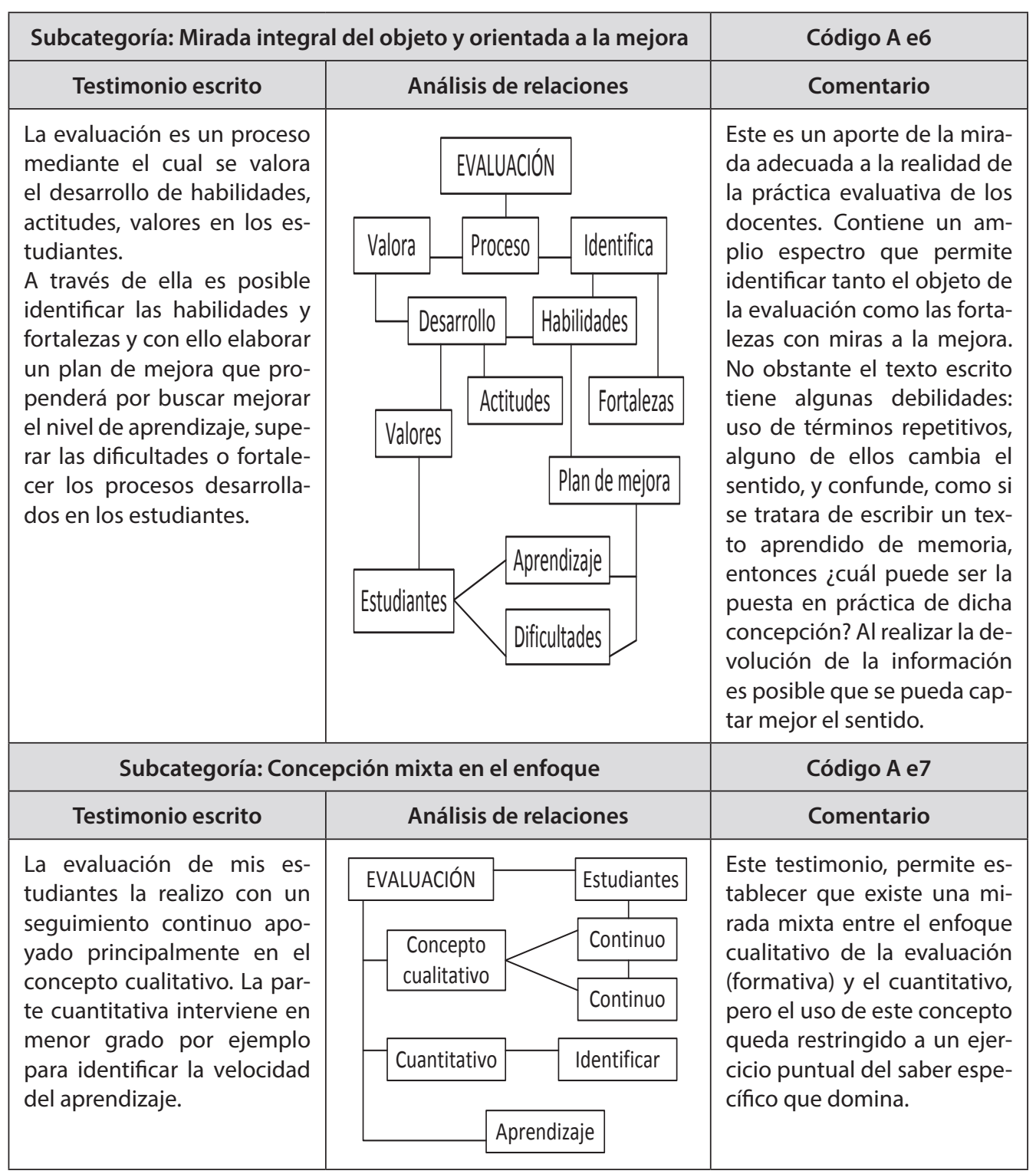


Álvaro Torres Mesías - Ana Barrios Estrada - Ruth Pantoja Burbano Los Sistemas Institucionales de Evaluación de los Estudiantes (SIEE) entre la teoría y la realidad práctica

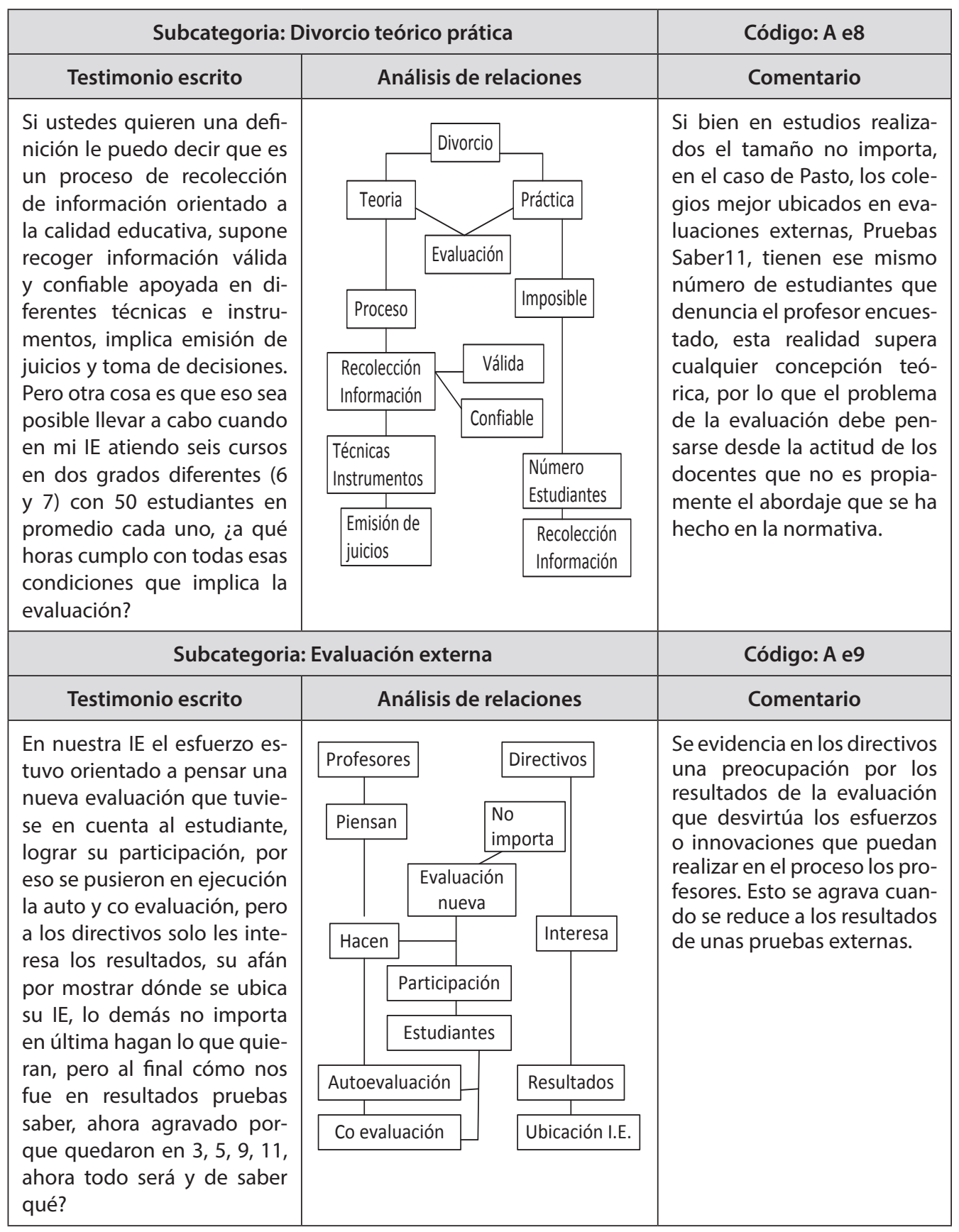

Fuente: Esta Investigación 


\section{a. Afectaciones e implicaciones del Decreto 1290/09}

\section{Cuadro 2: Principales afectaciones e implicaciones del decreto 1290/09}

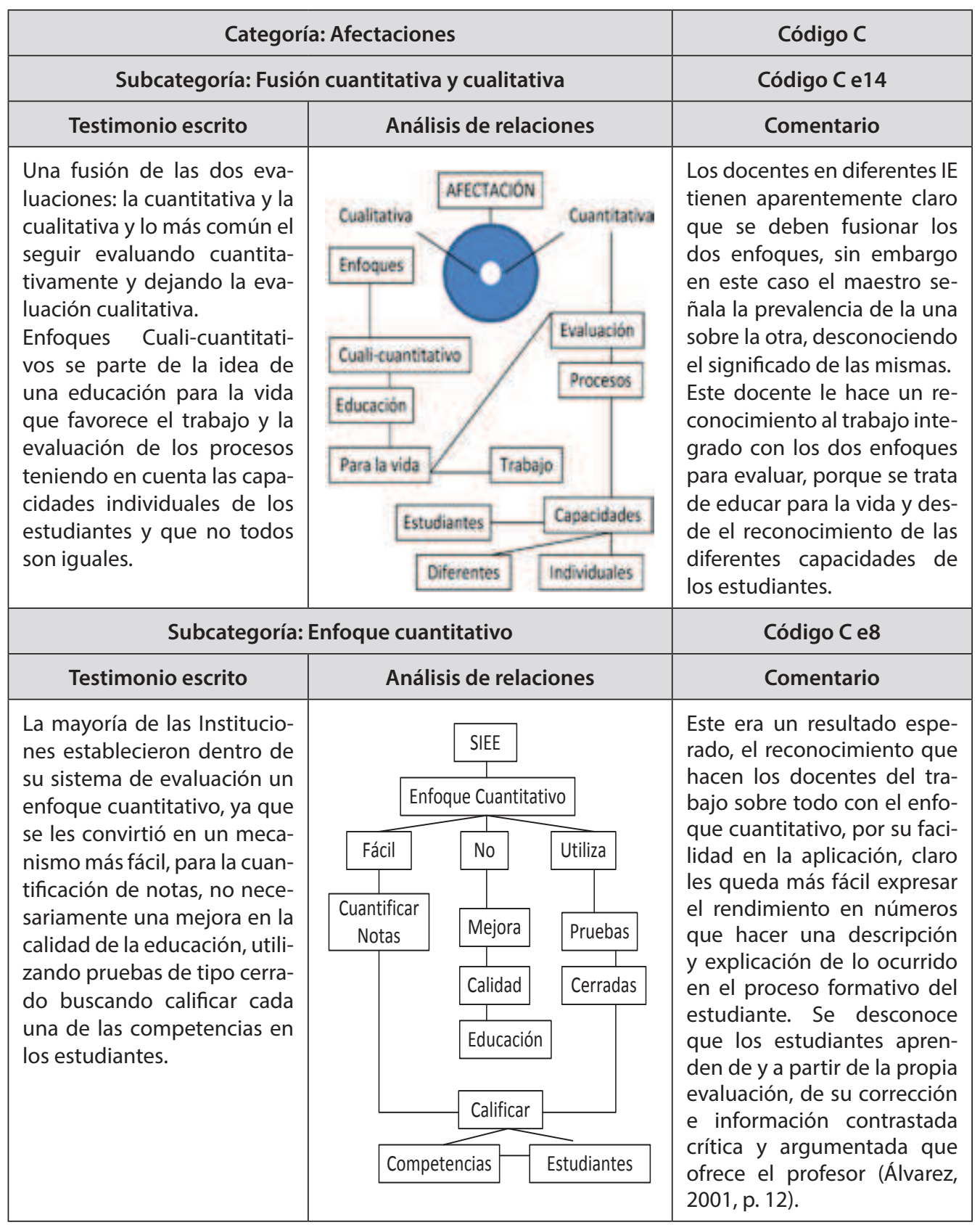


Álvaro Torres Mesías - Ana Barrios Estrada - Ruth Pantoja Burbano Los Sistemas Institucionales de Evaluación de los Estudiantes (SIEE) entre la teoría y la realidad práctica

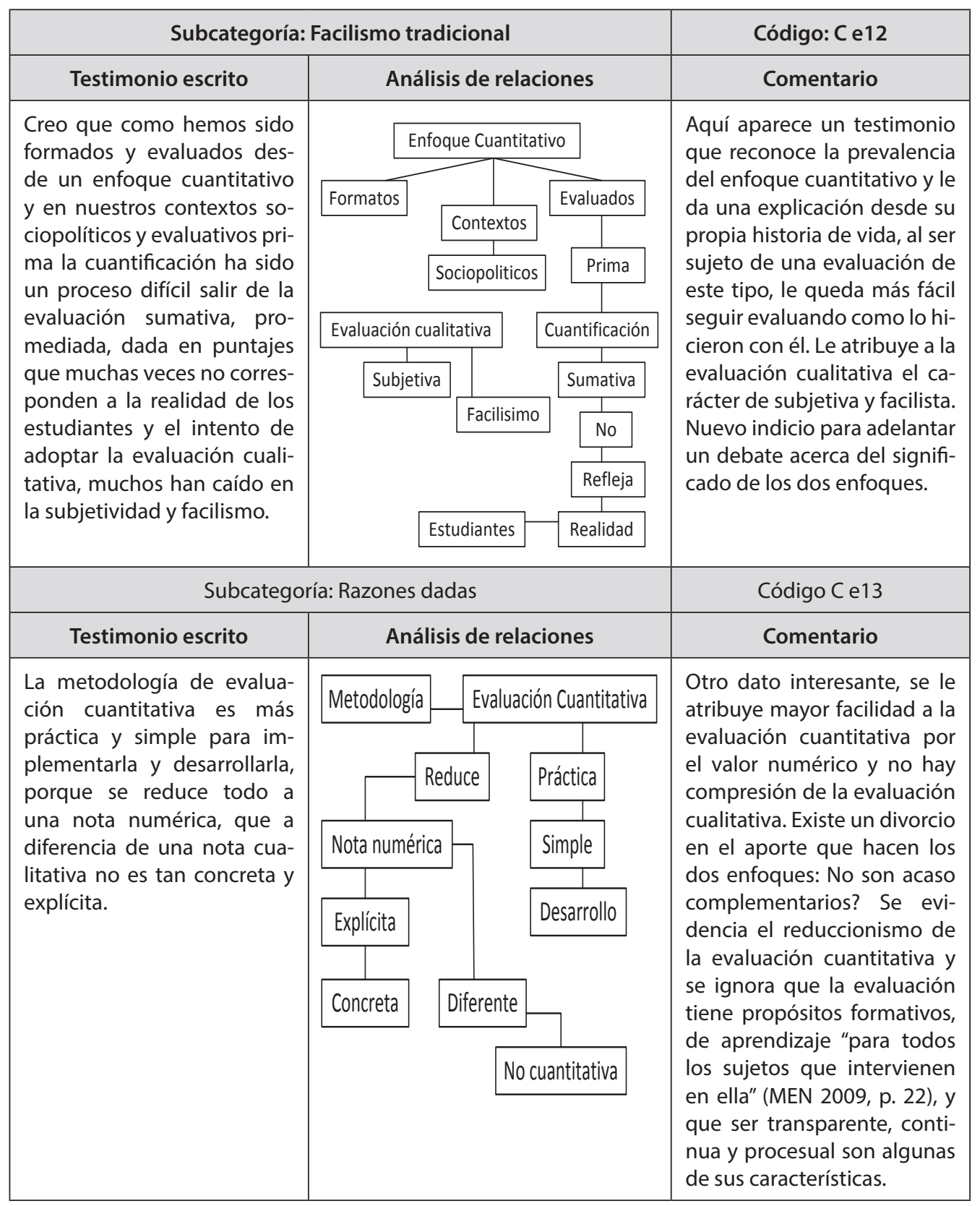


Revista TENDENCIAS Vol. XV No. 1

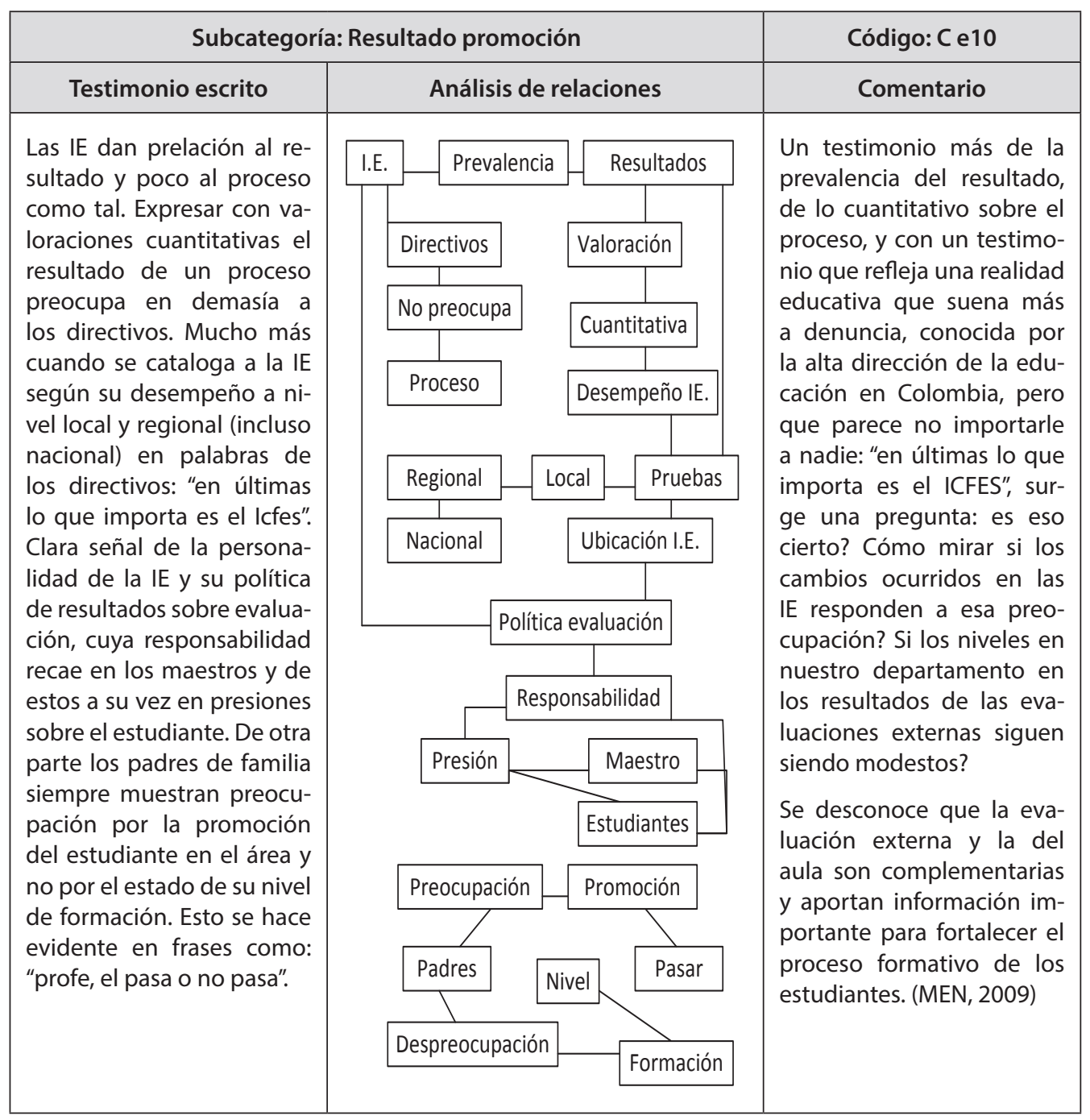

Fuente: Esta Investigación 


\section{b. Características de la evaluación formuladas en el SIEE}

\section{Cuadro 3: Características de la evaluación de las Instituciones Educativas objeto de estudio}

\begin{tabular}{|c|c|c|c|}
\hline \multicolumn{4}{|c|}{ CARACTERÍSTICAS DE LAS INSTITUCIONES } \\
\hline INSTITUCIÓN 1 & INSTITUCIÓN 2 & INSTITUCIÓN 3 & INSTITUCIÓN 4 \\
\hline $\begin{array}{l}\text { Integral, } \\
\text { Dialógica, Formativa }\end{array}$ & $\begin{array}{l}\text { Continua, } \\
\text { Integral, } \\
\text { Sistemática, } \\
\text { Flexible, } \\
\text { Interpretativa, } \\
\text { Participativa, } \\
\text { Formativa. }\end{array}$ & $\begin{array}{l}\text { Continua, } \\
\text { Integral, } \\
\text { Sistemática, } \\
\text { Flexible, } \\
\text { Participativa, } \\
\text { Formativa, } \\
\text { Enfocada a las compe- } \\
\text { tencias: Argumentati- } \\
\text { va, Propositiva, } \\
\text { Interpretativa } \\
\text { Enfocada a los desem- } \\
\text { peños. }\end{array}$ & $\begin{array}{l}\text { Continua, } \\
\text { Sistemática, } \\
\text { Flexible, } \\
\text { Interpretativa, } \\
\text { Participativa, } \\
\text { Formativa, } \\
\text { Integral }\end{array}$ \\
\hline
\end{tabular}

Fuente: Esta investigación

Como puede observarse, las características de la evaluación en la diferentes Instituciones objeto de estudio, tienen un listado que va de 3, que incluso son recurrentes en las instituciones estudiadas, a 7; y en una de ellas pasan de enumerarlas, a decir qué evalúan, lo que muestra la dispersión conceptual existente; esa pluralidad, hace que cuando se buscan evidencias de la puesta en práctica de dichas características, se torne difusa, por lo que hace más difícil entender las concepciones existentes en torno a la evaluación, y obliga a pensar en que este estudio debe ser objeto de otra investigación.

\section{PROPUESTA PARA UNA EVALUACIÓN ALTERNATIVA}

La alternativa estaría en la construcción de una "escuela evaluadora”, cuyo eje central curricular y pedagógico sea la evaluación que construye sujetos evaluadores: estudiantes y docentes, que invitan a otros sujetos: padres de familia y evaluadores externos, para generar una cultura evaluadora más allá de la norma, más allá de los discursos académicos externos que provocan mayor confusión y caos.

Sería una experiencia construida desde dentro, cuyos protagonistas son precisamente los diferentes sujetos que participan en la labor educativa y formativa: docentes, directivos, estudiantes, padres de familia que están implicados en todo proceso de evaluación, en su condición humana, al manifestar sentimientos, valores, creencias, concepciones sobre el aprendizaje, la enseñanza. 
Siendo la educación una práctica social y la evaluación uno de sus principales actos que se lleva a cabo en las instituciones educativas, que no se puede despojar de su función social, política y pedagógica (Santos, 2003).

La función social y política se relaciona con la promoción, con el fracaso escolar, con el éxito, con la deserción porque el proceso de enseñanza y aprendizaje se desarrollan en un clima que se caracteriza por los conflictos y la incertidumbre que están presentes y forman parte de las instituciones educativas. Mientras que la función Pedagógica: Diagnostica, selecciona, jerarquiza, comprueba, comunica, dialoga, forma, describe, explica, comprende, motiva, investiga, orienta, provoca el diálogo y la mejora, por tanto, la evaluación se relaciona con procesos fundamentales de la pedagogía.

Acogiendo los planteamientos de Santos (2003), se pretende superar la concepción de evaluación que se limita a discriminar, jerarquizar y calificar para avanzar hacia una comprensión de la evaluación, que nos permita aprender para mejorar la enseñanza de los profesores, el aprendizaje de los estudiantes y la calidad de las instituciones educativas.

En consecuencia se propone la evaluación de competencias Frola (2007) entendida como un proceso funcional sistemático, continuo, integral, orientador y cooperativo para obtener información sobre la manera en que el estudiante, demuestra ser competente a través de sus desempeños sobre los que se realiza una evaluación integral de competencias y habilidades del pensamiento, referidos a ciertos criterios -indicadores- para comprender el estado de los aprendizajes. Implica concebir la evaluación como proceso, que a lo largo del curso ha de proponer con cierta periodicidad actividades, de carácter evaluable, que faciliten la asimilación y desarrollo progresivos de los conocimientos, de las habilidades de pensamiento y competencias a alcanzar, respectivamente.

Se trata de aprender haciendo, de tal forma que la evaluación se convierte en un proceso continuo o progresivo, es eminentemente un proceso de investigación, con todas las características de ésta, mediante lo cual, el profesor puede realizar un mayor y mejor seguimiento del progreso en el aprendizaje del estudiante, ya que permite una valoración integral que responde a los siguientes interrogantes:

¿Para qué se evalúa?: Evaluación integral de competencias y habilidades del pensamiento.

¿Qué se evalúa?: Visión integral del aprendizaje y del desarrollo del individuo como ser humano.

¿Cuándo se evalúa?: Al inicio, durante y al final del proceso de aprendizaje.

¿Quién evalúa?: Autoevaluación, coevaluación, heteroevaluación

¿Cómo se evalúa? : Actividades evaluativas variadas, instrumentos y formas cualitativas. 
La evaluación de competencias comprende:

- El contexto o ambiente de aprendizaje y sus factores

- El saber o conocimiento

- Las competencias a desarrollar. Referentes

- Las actividades de evaluación

- Las herramientas de evaluación

Se relacionan a continuación algunas actividades e instrumentos que pueden contribuir a la evaluación cualitativa de competencias y habilidades de pensamiento.

\section{Cuadro 4. Actividades e Instrumentos de Evaluación}

\begin{tabular}{|c|c|c|}
\hline \multicolumn{2}{|c|}{ Actividades para evaluar competencias y habilidades de pensamiento } & \multirow{2}{*}{$\begin{array}{c}\begin{array}{c}\text { Herramientas para } \\
\text { evaluación cualitativa } \\
\text { de competencias }\end{array} \\
\text { Según Frola (2007) }\end{array}$} \\
\hline Según Delgado (2006) & Según Frola (2007) & \\
\hline Actividades de completación & Mapas mentales y conceptuales & La rúbrica \\
\hline Preguntas de desarrollo & Videos & La lista de chequeo \\
\hline \multirow{3}{*}{$\begin{array}{l}\text { El trabajo con textos y estudios com- } \\
\text { parativos: recensiones, "noticia o } \\
\text { reseña de una obra literaria o cientí- } \\
\text { fica," resúmenes, esquemas, cuadros, } \\
\text { gráficas, tablas }\end{array}$} & Programa de radio escolar & La escala estimativa \\
\hline & Periódico y noticiero escolar & \\
\hline & Debates & \\
\hline \multirow{2}{*}{$\begin{array}{l}\text { Elaboración de dictámenes, informes } \\
\text { y escritos (trabajo con formularios) }\end{array}$} & Carteles & \\
\hline & Exposiciones orales & \\
\hline Formulación de supuestos prácticos. & Collage & \\
\hline \multirow{2}{*}{$\begin{array}{l}\text { Búsqueda y recopilación de informa- } \\
\text { ción: elaboración de un dossier. }\end{array}$} & Frisos & \\
\hline & Proyectos & \\
\hline
\end{tabular}

Fuente: Esta investigación

\section{CONCLUSIONES}

Los hallazgos de esta investigación muestran como las percepciones y actitudes de los profesores, estudiantes y padres de familia que tienen sobre el SIEE y su aplicación en las Instituciones Educativas son diversas y algunas veces coinciden pero en otros aspectos difieren como se concluye en los siguientes párrafos. 
Se encuentra dificultades para comprender el SIEE y la nueva normativa, debido a que la evaluación es uno de los procesos más complejos, que presenta mayor resistencia al cambio en un acto educativo y en este caso específicamente está asociado a las nuevas demandas que se le hace al maestro, en el sentido de crear con autonomía su propio modelo de evaluación apoyado en la reflexión, la participación y el estudio que le permita implementar estrategias novedosas en éste campo que busquen la calidad educativa.

Frente a estas demandas algunos maestros una vez más evidencian la fuerte influencia que tiene sobre ellos el peso de la tradición que impide el cambio, cuando se crea una correspondencia entre el mejoramiento del rendimiento académico y la implementación del SIEE, de tal manera que se sostienen las estructuras académicas y administrativas acríticas, el divorcio entre teorías y prácticas, la evaluación en función solamente de los resultados desconociendo que en esencia el sentido y los propósitos de la evaluación se refieren sobre todo a las de obtener información sobre "aprendizajes significativos, críticos, autónomos, cooperativos, sociales y solidarios" (MEN, 2009: 29) de los estudiantes.

Es así que éstos maestros consideran que no se requiere mayor preparación para implementar el SIEE porque solo trata de mejorar el rendimiento académico, mientras los profesores que entienden y asumen el sentido y los propósitos de la nueva propuesta de evaluación consideran que les hace falta preparación.

Un hallazgo importante permite señalar que algunos docentes tienen aún, una mirada instrumental de la evaluación, ya que como se registró arriba, "el propósito de la evaluación es apropiarse de herramientas, en un caso y en el otro que la evaluación es una herramienta de trabajo", concepción presente en los imaginarios de los docentes que se debe erradicar por su carácter instrumental y superarse con una sólida fundamentación teórica y metodológica.

Uno de los puntos a resaltar es la resistencia que un grupo de docentes expresa ante la concepción sobre evaluación, que en una correcta aplicación estaría entre las más pertinentes: "supone recoger información válida y confiable apoyada en diferentes técnicas e instrumentos, implica emisión de juicios y toma de decisiones". No obstante un docente considera que esto no es posible llevar a cabo, debido a que en su I.E. trabaja con seis cursos, en dos grados diferentes $\left(6^{\circ}\right.$ y $\left.7^{\circ}\right)$ con 50 estudiantes en promedio por cada curso, por tanto manifiesta: "¿a qué horas cumplo con todas esas condiciones que implica la evaluación?”. Esta realidad supera cualquier concepción teórica, por consiguiente el problema de la evaluación debe pensarse desde la actitud de los docentes que no es propiamente el abordaje que se ha hecho en la normativa, que puede estar bien concebida, pero las concepciones y prácticas evaluativas de los maestros pueden desvirtuar lo pretendido en dichas normativas.

Otra evidencia está en el papel que juegan los directivos, al preocuparse excesivamente por los resultados de las evaluaciones externas, lo cual desvirtúa los esfuerzos o innovaciones que puedan realizar los profesores en el proceso. Esto se agrava cuando toda la tarea institucional se reduce a los resultados de las pruebas 
como se registra en el siguiente testimonio: "En nuestra IE el esfuerzo estuvo orientado a pensar una nueva evaluación que tuviese en cuenta al estudiante, lograr su participación por eso se pusieron en ejecución la auto y co evaluación, pero a los directivos solo les interesa los resultados, su afán por mostrar dónde se ubica su Institución Educativa, lo demás no importa.”

Por otra parte, es necesario y conveniente señalar -para no caer en miradas absolutistas-, que se identificaron maestros e instituciones educativas con claros indicios de su avance hacia nuevos escenarios de evaluación formativa y continua de los aprendizajes desde enfoques incluyentes, integradores, participativos para el desarrollo de las competencias, con la intencionalidad de superar la preocupación exclusiva por el rendimiento académico y la idea de evaluar para medir, calificar el dominio de contenidos como resultado de la transmisión de conocimientos. Entre esos indicios se pueden registrar:

- Los criterios de evaluación son claros y negociados entre profesores y estudiantes.

- Se realiza un acompañamiento para que el estudiante logre nivelarse en cuanto a las competencias y estándares establecidos para determinado grado.

- Se ejerce la autonomía para determinar la promoción de los estudiantes y el uso de los resultados de la evaluación para el ajuste e implementación del plan de mejoramiento institucional, en busca de la calidad.

- Se promueve la integración entre enseñanza, aprendizaje y evaluación como procesos importantes, continuos y articulados a la formación. 


\section{REFERENCIAS}

ÁLVAREZ MÉNDEZ, J. M. (2001). Evaluar para conocer, examinar para excluir. Madrid: Morata.

CANO, M.E. (2008). La evaluación por competencias en la educación superior. En: Revista Profesorado. Revista de Curriculum y formación del profesorado. Barcelona.

DELGADO, A. M. (2006). Evaluación de las competencias en el espacio europeo de educación superior. Barcelona: Boch Ediciones

DELORS, J. (1998). Informe a la Unesco de la comisión internacional sobre la educación para el siglo XXI: La educación encierra un tesoro. Madrid.

FROLA, P. (2007). Maestros competentes a través de la planeación y la evaluación por competencias. Trillas, México.

GARCÍA, J. (2003). Didáctica de las ciencias. Resolución de problemas y desarrollo de la creatividad. Bogotá: Magisterio.

MINISTERIO DE EDUCACIÓN NACIONAL (2009). Decreto 1290 de 2009. República de Colombia.

MINISTERIO DE EDUCACIÓN NACIONAL (2009). Fundamentos y orientaciones para la fundamentación en la implementación del Decreto 1290 de 2009. República de Colombia.

GUERRA, M. Á. S. (2003). Una flecha en la diana: la evaluación como aprendizaje (Vol. 166). España: Narcea Ediciones.

STUFFLEBEAM, D. (2002). Evaluación Sistémica: Guía teórica y práctica. España: Ed. Paidós Ibérica, S.A.

TOBÓN, S. (2005). Formación basada en competencias: pensamiento complejo, diseño curricular y didáctica. Bogotá: Ecoe Ediciones.

TORRES, M. A. y Otros (2002). Las prácticas evaluativas de los maestros de la E.N.S. de Nariño y Putumayo. U DE N. Pasto.

TORRES, M. A. (2006). Análisis histórico lógico de la evaluación en Colombia período 1967-2006. Ponencia Congreso Internacional de Educación. Rudecolombia. Paipa, Boyacá.

TORRES, M. A. (2009). Evaluación Democrática. En: Maestro Legal, Pasto. 